\title{
Development and essence of global evolutionism problem
}

\author{
Olga Brusko* \\ Novorossiysk branch of the federal state budgetary educational institution of higher education \\ "Belgorod State Technological University named after V.G. Shukhov", 353900, Novorossiysk, Russia
}

\begin{abstract}
The purpose of this article is to disclose ideas about the development and essence of global evolutionism problem. The author claims that global evolutionism is, on the one hand, one of the fundamental principles of the reality existence, but at the same time an integrative research concept, built on a universal model of evolution, synthesizing the development of space, earth, nature, society and the individual person. The development of global evolutionism concept was facilitated by the ideas of traditional for philosophy processuality and development, which, however, were ambiguously interpreted in the history of culture. Global evolutionism is based on a systematic study of nature through a theoretical synthesis of evolutionary and systemic research methods. Global evolutionism development is a vivid proof that by now philosophy has not lost its ability to generate new areas of not only separate science, but also of general scientific and interdisciplinary knowledge.
\end{abstract}

\section{Introduction}

The global evolutionism problem is one of the leading problems for modern philosophy, which is of great importance and relevance in all historical eras of the human culture development. In the XXI century, it became clear that evolutionary transformations are much larger, wider, deeper and more global than was traditionally considered not only within the scientific community, but also in everyday consciousness. All this gives rise to the need for a deep and comprehensive, holistic and multifaceted study of the global evolutionism problem. Global evolutionism is, on the one hand, one of the fundamental principles of the existence of reality, but at the same time, an integrative research concept, built on a universal model of evolution, synthesizing the development of space, earth, nature, society and an individual. This definition, obviously, divides the concept of global evolutionism into two aspects: ontological reality, the most important property of being, its mode of existence and epistemological approach, research direction. At the same time, most researchers interpret global evolutionism in the second sense, that is, not as a universal ontological principle, but as a concept. The development of global evolutionism concept in the history of culture was facilitated by the ideas of processuality and development.

\footnotetext{
* Corresponding author: olya.swee2014@yandex.ru
} 


\section{The idea of processuality in the light of global evolutionism concept development}

One of the fundamental characteristics of nature is its processuality, which permeates all the facets and forms of the manifestation being. Initially, the idea of processuality was present in the philosophy of the East and Antiquity in the form of an idea of events and phenomena circulation in reality. At the same time, such concepts as "wheel", "circle", "rotation" were used, which emphasized not only the variability of the existing, but constant returns to previously passed states of the phenomenon. Therefore, ancient man still does not distinguish between the concepts of "past", "present" and "future", does not understand and does not recognize the irreversibility and inevitability of historical events: "Look at the cycle of things again hurrying to the past: you will see that nothing in this world is destroyed, but it only sets and rises again" (Seneca, 1977, p. 65). This implies that the ontology of antiquity practically does not contain the problems of development and time.

The advent of the Christian era marked the emergence of the time problem in the human culture history. However, the religious vision of the world gives the perception of time a mystical character as a God-preset movement of history. The past and the future are explicated as a manifestation of the present: “... the present of the past is memory, the present of the present is its direct contemplation, and the present of the future is its expectation" (Augustine, 1991, p. 297). This implies that time is interpreted linearly as a directed course of events that exists, from the view point of Augustine, only in the human mind. At the same time, one cannot fail to note that medieval culture and philosophy do not pay due attention to nature, which was of interest in the context of the interpretation and decoding of religious allegories. As N. A. Berdyaev correctly noted, "Christianity tightly closes the inner life of nature and does not let man into this life" (Berdyaev, 1990, p. 90). Thus, in spite of the emergence of the time idea, it did not belong to nature, but, naturally, man was primarily considered as an image and likeness of God, and not a being, related to nature.

The ideas of close attention to nature, the idea of openness and incompleteness of the Universe, the idea of earthly life value and man recognition as creative, but at the same time divine creation came to culture during the Renaissance era.

In Modern times, the idea of movement and processuality in nature for the first time becomes an independent subject of study. Moreover, the movement in nature, according to F. Bacon, is hidden from a man at the level of sensory experience and can only be known experimentally, which allows you to make "... a discovery in every generation and movement of a hidden process that continues infinitely from the manifested acting principle and the manifest matter down to this form ... "(Bacon, 1972, p. 83). In turn, this requires a person to achieve power over nature, knowledge about the variability of nature.

In the last quarter of the XIX century, the idea of process arises as a cognition form, which was characterized by: "a) a regular connection and a successive changes of phases, cycles, states of the whole; b) temporary (genetic) difference between the changing states of the object; c) the irreversible nature of qualitative changes " (Keligov, 2012, p. 16). Of course, one should not assume that this idea has received widespread acceptance and dissemination. However, in many subject areas of knowledge, this idea has become a research principle.

\section{The idea of development in the context of global evolutionism concept essence}


The idea of the processuality in nature is closely connected with the idea of development, which penetrated the scientific consciousness in the middle of the XVIII century and was associated with the need for an objective and temporary measurement of phenomena from their occurrence to extinction. The key role in the study of man and nature as developing processes was played by I. Kant's nebular hypothesis (Kant, 1963) about the origin of the solar system and the theory of K.F. Wolf's epigenesis (Wolf, 1950) on the gradual embryo development, after which developing processes have become the subject of special research in science.

The credit for creating a philosophical concept of development belongs to G.V.F. Hegel, who not only thought dialectically, not also considered the development idea as methodological principle, and specially, comprehensively and systematically studied it. $\mathrm{He}$ argued that philosophy itself "... is a knowledge of this development, and as thinking through concepts, it itself is this thinking development" (Hegel, 1932, p. 32). At the same time, Hegel acknowledges the otherness as the source of any development of thinking that determines the development of nature, self-constructing its own path and self-conscious Absolute idea, which essentially mystifies the dialectic science.

An alternative version of the general development theory is given in Marxism, where the idea of development is presented as an objective and universal regular process. In this case, the nature of universality is first of all the relations and connections between objects, things and phenomena of being that are originally generated by matter, which has the property of self-development. Marxism affirms that processes in nature and human society are only different phases of a single process for historical development. From this follows the recognition of human consciousness origin due to historical and genetic transformations of inorganic and organic nature, i.e. matter "comes to the development of thinking beings by virtue of its very nature, and therefore it necessarily occurs in all cases when there are relevant conditions (not necessarily everywhere and always the same)" (Marx, 1961, p. 524 ) At the same time, the Marxist interpretation of the development idea was not without flaws, because it contained a revolutionary ideological component, which hardly contributed to the deepening of the scientific community in the problem of studying man and nature as a developing process.

The emergence of synergetics in the XX century marked a transition not only to the postulation of the processuality idea, but to the disclosure of the empirical foundations of globality and the inclusiveness of this idea, which was made possible by proving the existence of open and non-equilibrium systems in all spheres of life. From the point of view of synergetics, the surrounding world is not perceived as a museum, it "... is rather a world of processes in which information and structure are destroyed and saved" (Prigogine, E. Roerich, N. Roerich, 1991, p. 35). In other words, our world is a series of creative and destructive processes in which the attributive development is specifically universal in nature and manifests itself in the form of a natural principle of life of self-organizing objects in the framework of interacting systems. Development is manifested in the form of a translational process of self-organizing systems transformation, in which one of the opposite trends dominates during the transition from one substantial level to another.

\section{Genesis and essence of global evolutionism concept}

The origins of global evolutionism concept should be sought in the birth of the very idea of evolution (from lat. Evolutio - deployment). Even in Buddhism, you can find the idea of "pratya-samutpada", interpreted as a dependent principle, conditional reproduction and interconnection: "According to Buddhism, the world consists of an infinite number of interdependent qualities that manifest themselves in time and space under certain conditions as part of a whole. Any change in the universe entails inevitable consequences, because all 
that exists is in close interconnection" (Rudenko, 2018, p. 39). Three signs of universe existence follow from this idea in the philosophy of Buddhism: anigga, anatta and dukkha. The first of them is anigga, i.e. inconstancy and variability of the Universe - the essence of the evolution concept is consonant.

In ancient philosophy, the idea of evolution was first expressed by the representative of the Milesian school, the pupil of Thales, Anaximander, who believed that "man came about as a result of evolution from other animals ..." (Rudenko, 2012, p. 56), which actually anticipated the teachings of C. Darwin. Among the other prominent thinkers of antiquity who played a large role in shaping the idea of evolution, we should single out Heraclitus of Ephesus, who was convinced that "everything flows, everything changes" and that "you cannot enter the same river twice", and also brought the key law of dialectics that the transition of a phenomenon from one state to another is accomplished through the struggle of opposites.

Evolutionary processes began to attract more attention of researchers, philosophers and scientists in the XVIII - XIX centuries. In this regard, it is worth mentioning the KantLaplace nebular hypothesis about the origin of the solar system from a nebula, C. Lyell's idea of the geological evolution of the earth's layers, and finally, C. Darwin's theory of the species origin by natural selection.

The development of global evolutionism concept, which was shaped in the $80 \mathrm{~s}$ of the $\mathrm{XX}$ century, was facilitated by "the theory of the unsteady Universe, the concept of the biosphere and noosphere, as well as the ideas of synergetics" (Leshkevich, 2005, p. 180). Within the framework of global evolutionism, many ideas that were previously considered true were rethought, including many of the principles of Charles Darwin's evolutionary theory.

Relativistic cosmology has become a determining factor in creating the concept of global evolutionism. Discoveries of extragalactic astronomy, including the discovery of the "scattering of galaxies" by E. Hubble (the redshift phenomenon of distant galaxies) in 1929 and the proof in 1922 by A.A. Friedman of the periodic expansion or contraction of the Universe based on the analysis of the equations of the general relativity theory by A. Einstein became the empirical basis of the hypothesis of Universe cosmic evolution.

After the concept of "open system" appeared in synergetics, it was possible to eliminate the contradiction that existed between the understanding of evolution in physics (for example, classical thermodynamics) and biology (for example, in the evolutionary theory of C. Darwin): "If, according to the second law of thermodynamics, entropy is a measure of disorder and disorganization in closed systems of inorganic nature is constantly increasing, then in living systems evolution, on the contrary, is accompanied by an increase in order and an improvement in their organization" (Ruzavin, 2008, p. 16). Only in non-equilibrium thermodynamics and synergetics, based on a new understanding of an open system, constantly exchanging energy and matter with the environment, as well as information, could this contradiction be eliminated, and the concept of a closed system should be discarded as an excessive abstraction that reduces reality. Moreover, such concepts as "equilibrium", "steadiness", "stability", were rethought. They began to be perceived only as a certain step or level in the overall development process. And all development ends with the emergence of a new one. Thus, any open system has a non-equilibrium and unstable character, which is the most important prerequisite for analyzing the evolution of a complex system, which makes a significant contribution to understanding the essence of global evolutionism.

In global evolutionism, the concept of "globality" is understood not so much in Latin (from Latin globus - sphere), but in French (from French global - universal, taken as a whole) meaning, wider in meaning, i.e. extending not only to the planet, but to the entire universe. 
Academician of the World Ecological Academy, Doctor of Philosophical science, professor G.I. Ruzavin notes that global evolutionism is now understood as "a direction of research focused on representing the world around us and the Universe as a single system of interacting evolutionary systems" (Ruzavin, 2008, p. 16). At the same time, he emphasizes that this theory has not yet been completely constructed, that this is a matter for the near future, and that further development of the cosmic evolution principles, a systemic method, and synergetics is necessary for this.

Representing the whole world as a huge evolving system, the concept of global evolutionism has universality, great integrative potential, and also emphasizes the idea of the unity for the universe, which is constantly evolving. As notes T.G. Leshkevich, "global evolutionism includes four types of evolution: cosmic, chemical, biological and social, combining them with genetic and structural continuity" (Leshkevich, 2005, p. 180). Therefore, this concept has a methodological potential that allows for the integration of science, socio-humanitarian and technical knowledge, which opens up significant opportunities for researching with its help and in its context human problems from philosophical and anthropological positions. But at the same time, global evolutionism is interesting not only from epistemological positions, but also in the ontological aspect, allowing us to consider man in the context of the constant and natural development not only nature, but also culture.

Global evolutionism is based on a systematic study of nature through a theoretical synthesis of evolutionary and systemic research methods. This opens up the possibility of explicating the genesis of the Universe in its integrity and internal interconnection, interdependence and interaction. Calling the concept of global evolutionism one of the most fundamental and promising research projects, A.D. Ursul and T.A. Ursul name it as "... an integrative, general scientific and transdisciplinary approach to understanding basically the progressive development of matter, which differs significantly from the picture of the previous vision of evolutionary processes" (A.D. Ursul, T.A. Ursul, 2015, p. 45). Whereas earlier concepts of development relied on individual research results within the framework of specific scientific disciplines, the concept of global evolutionism has an integrative general scientific, transdisciplinary status. And the very development of global evolutionism is vivid proof that at present philosophy has not lost the ability to generate a new field of not only private science, but also general scientific and interdisciplinary knowledge.

\section{Research methods}

The methodological and theoretical basis of this study was composed not only of the works of scientists and philosophers, thanks to whom it was possible to reveal the development of the idea of processuality and development, which subsequently formed the basis for the emergence of the concept of global evolutionism, but also modern researchers. Among the first, the works of Seneca and St. Augustine, F. Bacon, I. Kant, G. Hegel, K. Marx, C. Darwin, N. Berdyaev should be mentioned first. I. Prigogine, K.F. Wolf et al. Among modern researchers, the works of A.D. Ursul, D.A. Ursul, K. Sagan, G.I. Ruzavina, M.Yu. Keligova, T.G. Leshkevich, A.M. Rudenko et al make a great contribution to the study of the problem essence of global evolutionism.

During work process, a phenomenological-hermeneutic approach was used, with the help of which the author carried out both understanding and explanation of the analyzed scientific and philosophical texts. In addition, the study also used a systematic approach, with the help of which it became possible to analyze the problem of the global evolutionism essence and development. 


\section{Conclusion}

Thus, the study allows us to draw a number of conclusions.

Global evolutionism is, on the one hand, one of the fundamental principles of the reality existence, but at the same time, an integrative research concept, built on a universal model of evolution, synthesizing the development of space, earth, nature, society and individual. The concept of global evolutionism is divided into two aspects: ontological reality, the most important property of being, its mode of existence and epistemological approach, research direction. At the same time, most researchers interpret global evolutionism in the second sense.

The idea of the movement and processuality in nature for the first time becomes an independent subject of study in modern times. The credit for creating a philosophical concept of development belongs to G.V.F. Hegel, who not only thought dialectically, not only considered the idea of methodological principle development, but also specially, comprehensively and systematically studied it. An alternative version of the general theory of development is given in Marxism, where the idea of development is presented as an objective and universal regular process and it is stated that processes in nature and human society are only different phases of a single process of historical development. The concept of synergetics in the XX century contributed to the discovery of the empirical foundations of globality and the inclusiveness of this idea, which was made possible by proving the existence of open and non-equilibrium systems in all spheres of life. The development of global evolutionism concept, which took shape in the 80s of the XX century, was facilitated by: the theory of the unsteady Universe, the concept of the biosphere and noosphere, as well as the ideas of synergetics. Within the framework of global evolutionism, many ideas that were previously considered true were rethought, including many of the principles of Charles Darwin's evolutionary theory. Relativistic cosmology has become a determining factor in creating the concept of global evolutionism.

In global evolutionism, the concept of "globality" is understood to extend not only to the planet, but also to the entire Universe. Global evolutionism is a field of research that represents the world and the Universe as a single system of interacting evolutionary systems. Representing the whole world as a huge evolving system, the concept of global evolutionism has universality, great integrative potential, and also emphasizes the idea of the unity of the universe, which is constantly evolving. This concept has a methodological potential that allows for the integration of science, socio-humanitarian and technical knowledge. Global evolutionism is based on a systematic study of nature through a theoretical synthesis of evolutionary and systemic research methods. The development of global evolutionism is vivid proof that at present philosophy has not lost the ability to generate a new field not only in private science, but also in general scientific and interdisciplinary knowledge.

\section{References}

1. A. Augustine Confession of Blessed Augustine, Bishop of Hippo, Per. with lat. M.K. Sergienko. Will enter. article A.A. Stolyarova (Publishing house "Renaissance",M ., 1991)

2. N.A. Berdyaev, The meaning of the story (Mysl', M., 1990)

3. F. Bacon Works: in 2 volumes, T.2. (T Mysl', M ., 1972)

4. K.F. Wolf, Theory of Origin. General edition of academician E.N. Pavlovsky (USSR Academy of Sciences,M ., 1950) 
5. Hegel G.V.F., Lectures on the history of philosophy. Book One, Collected Works. In 14 volumes. Volume 9. (Party publishing house, M., L ., 1932)

6. I. Kant Universal, Natural History and Theory of Heaven, Works in six volumes. Under the general editorship of V.F. Asmus. T. 1. (Mysl', M., 1963)

7. M.Yu. Keligov, Homo sapiens: A Transient Phenomenon (Academic Project; Culture, M ., 2012)

8. T.G. Leshkevich, Philosophy of Science: Textbook, (INFRA-M, M., 2005)

9. K. Marx, F. Engels, Compositions. Second Edition, T. 20 (Publishing house of political literature, M. 1961)

10. I. Prigogine, E. Roerich, N. Roerich, Nature, science and the new rationality // In search of a new worldview. M., Znanie, 7 (1991).

11. G.I. Ruzavin, Global evolutionism, Scientific notes of the Russian State Social University, 1 (57), (2008).

12. A.M. Rudenko, Philosophy in diagrams and tables. Ed. 3rd (Phoenix, Rostov-on-Don, 2018)

13. K. Sagan, Space: The evolution of the universe, life and civilization (Amphora, SPb., 2008)

14. Seneca, Moral letters to Lucius (Nauka, M. 1977)

15. A.D. Ursul, T.A. Ursul, The concept of universal evolutionism and global research, Materials of the annual Moses readings, 4, (2015) 